\title{
Severe Osteomalacia with Dent Disease Caused by a Novel Intronic Mutation of the CLCN5 gene
}

\author{
Ayumi Matsumoto ${ }^{1}$, Isao Matsui ${ }^{1}$, Takayasu Mori ${ }^{2}$, Yusuke Sakaguchi ${ }^{3}$, Masayuki Mizui ${ }^{1}$, \\ Yoshiyasu Ueda ${ }^{1}$, Atsushi Takahashi ${ }^{1}$, Yohei Doi ${ }^{1}$, Karin Shimada ${ }^{1}$, Satoshi Yamaguchi ${ }^{1}$, \\ Keiichi Kubota ${ }^{1}$, Nobuhiro Hashimoto ${ }^{1}$, Tatsufumi Oka ${ }^{1}$, Yoshitsugu Takabatake ${ }^{1}$, \\ Eisei Sohara ${ }^{2}$, Takayuki Hamano ${ }^{3}$, Shinichi Uchida ${ }^{2}$ and Yoshitaka Isaka ${ }^{1}$
}

\begin{abstract}
:
We present a case of Dent disease caused by a novel intronic mutation, 1348-1G $>$ A, of the chloride voltage-gated channel 5 (CLCN5) gene. Cultured proximal tubule cells obtained from the patient showed impaired acidification of the endosome and/or lysosome, indicating that the 1348-1G $>$ A mutation was indeed the cause of Dent disease. Although the prevalence of osteomalacia in Dent disease is low in Japan, several factors-including poor medication adherence-caused severe osteomalacia in the current case. Oral supplementation with calcium and native/active vitamin D therapy, with careful attention to medication adherence, led to the improvement of the patient's bone status.
\end{abstract}

Key words: Dent disease, osteomalacia, CLCN5, intronic mutation

(Intern Med 57: 3603-3610, 2018)

(DOI: 10.2169/internalmedicine.1272-18)

\section{Introduction}

Dent disease is a rare $\mathrm{X}$-linked, recessively inherited, proximal tubulopathy characterized by low-molecular-weight proteinuria (LMWP), hypercalciuria, nephrocalcinosis, and progressive renal failure (1). Approximately $60 \%$ of Dent disease cases are caused by mutations of the chloride voltage-gated channel 5 (CLCN5) gene, which encodes $\mathrm{H}(+) / \mathrm{Cl}(-)$ exchange transporter 5 (ClC-5) (2). In the proximal tubules of the kidney, $\mathrm{ClC}-5$ plays an important role in endosomal acidification and recycling, thereby controlling the reabsorption of solutes in the tubule lumen (3). In contrast to the high prevalence of LMWP (100\% of cases) and nephrocalcinosis (79\% of cases), the prevalence of osteomalacia is relatively low (33\%) in European patients with CLCN5 mutations (4). Moreover, Sekine et al. reported that osteomalacia was not observed $(0 \%$; 0 of 61 patients) in Japanese patients with CLCN5 mutations (5). We herein re- port a case of severe osteomalacia accompanied by Dent disease. Genomic DNA sequencing revealed a previously unrecognized intronic mutation of the CLCN5 gene that results in C-terminal truncation of $\mathrm{ClC}-5$.

\section{Case Report}

A man in his 40s was admitted to our hospital for the induction of peritoneal dialysis therapy for treatment of endstage renal disease (ESRD). He was healthy at birth, followed normal developmental milestones as a child, and ate a balanced diet. In his childhood, a medical checkup revealed evidence of urinary protein. At 19 and 20 years of age, he suffered from colic due to ureteral stones. His brother was also diagnosed as having ureteral stones (Fig. 1). There was no other family history of kidney disease (Fig. 1). Although no assessment was made to detect aminoaciduria in the 11 years prior to admission, the presence of proteinuria, glucosuria, and high urinary $\beta 2$-microglobulin suggested Fanconi

${ }^{1}$ Department of Nephrology, Osaka University Graduate School of Medicine, Japan, ${ }^{2}$ Department of Nephrology, Graduate School of Medical and Dental Sciences, Tokyo Medical and Dental University, Japan and ${ }^{3}$ Department of Comprehensive Kidney Disease Research, Osaka University Graduate School of Medicine, Japan

Received: March 27, 2018; Accepted: May 23, 2018; Advance Publication by J-STAGE: August 10, 2018

Correspondence to Dr. Isao Matsui, matsui@kid.med.osaka-u.ac.jp 


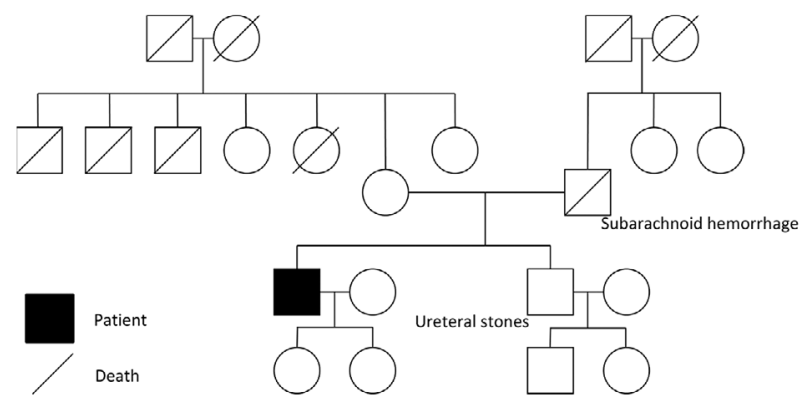

Figure 1. The family tree of the current case. The family tree of the current case. With the exception of his brother, no other members of the patient's family had a history of kidney disease. syndrome (Fig. 2A). His serum phosphate levels from 11 to 3 years prior to admission were low, whereas his serum creatinine levels were elevated. High fractional excretion of phosphate $\left(\mathrm{FE}_{\mathrm{P}}\right)$ at admission $(61.3 \%)$ also supported the diagnosis of Fanconi syndrome (6). His serum calcium levels gradually decreased, while his serum creatinine and alkaline phosphatase (ALP) levels increased during the 4 years prior to admission (Fig. 2A). His bone mineral density (BMD), as assessed by dual-energy X-ray absorptiometry, gradually decreased from 137 months to 50 months prior to admission (Fig. 2B). There was an exaggerated decline in the patient's BMD over the 4 years prior to admission, in keeping with the progression of hypocalcemia during this time (Fig. 2B). Calcitriol $(0.25 \mu \mathrm{g} /$ day $)$ was prescribed for several years prior to admission. As the patient was normotensive, no antihypertensive agents, including angiotensin-converting en-
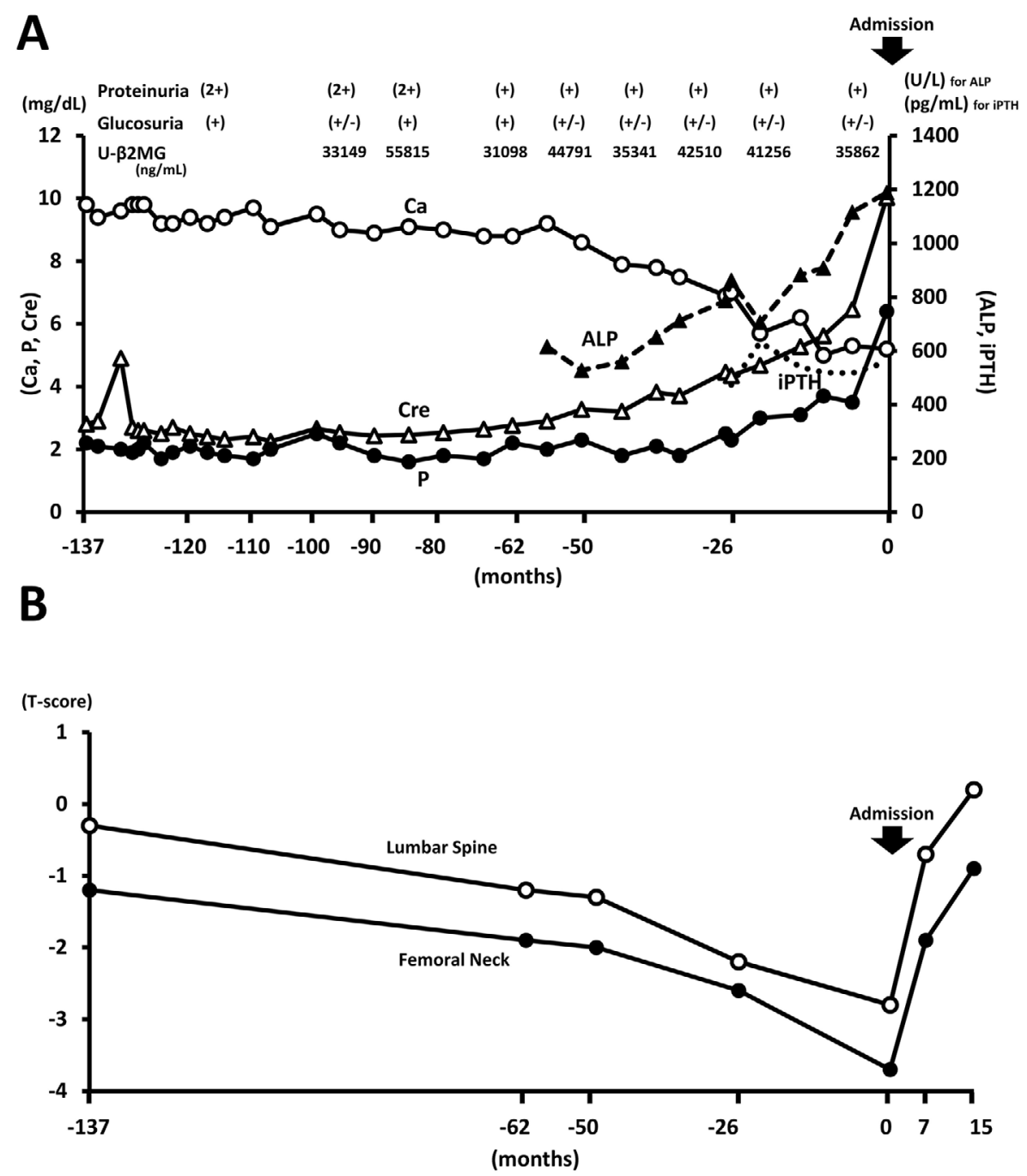

Figure 2. The clinical features of the current case. (A) The proteinuria, glucosuria, urinary $\beta 2$ microglobulin, serum phosphate, calcium, alkaline phosphatase, intact PTH, and creatinine levels during the 11 years prior to admission are shown. (B) Dual-energy X-ray absorptiometry of the lumbar spine and femoral neck on admission revealed an extremely low bone mineral density. ALP: alkaline phosphatase, Ca: calcium, Cre: creatinine, P: phosphate, PTH: parathyroid hormone,

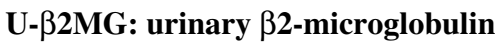


A

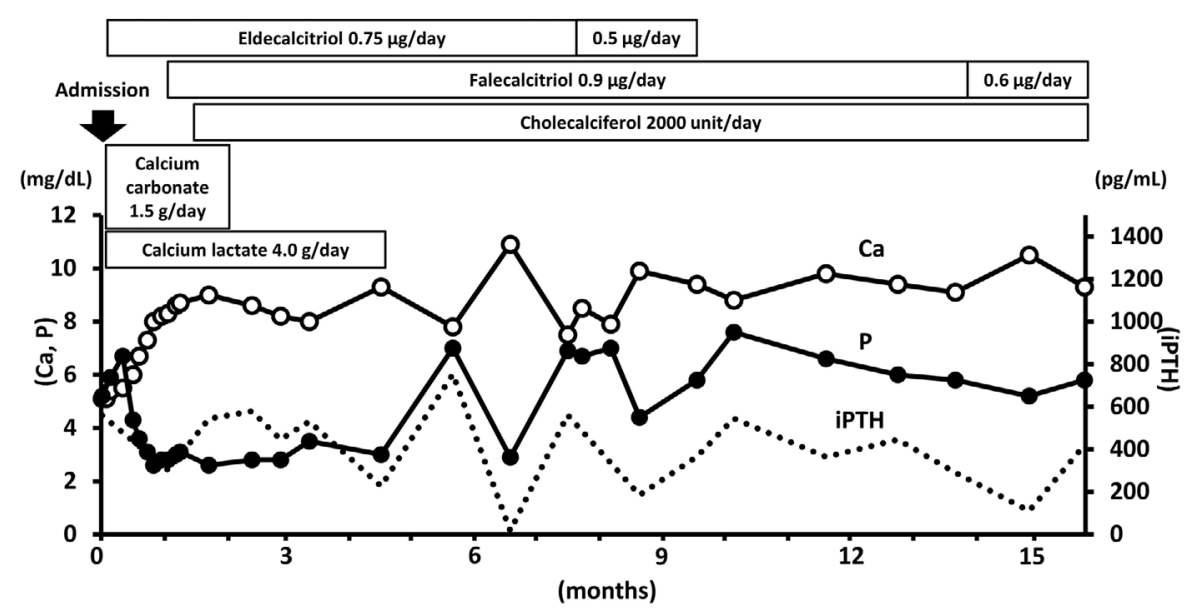

B

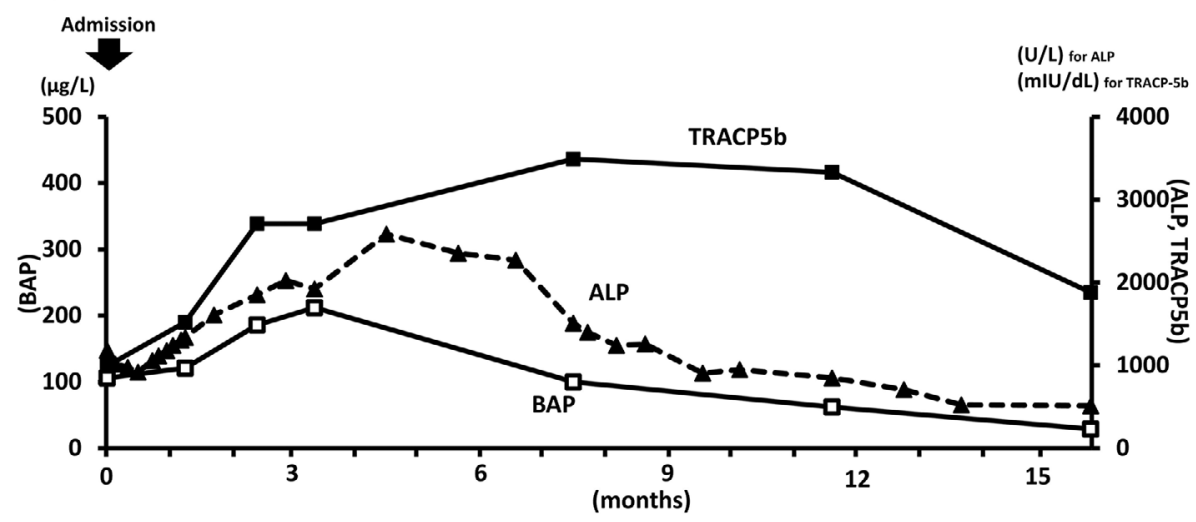

Figure 3. The therapeutic process in the current case. (A) A summary of the therapeutic process is shown. (B) The changes in bone metabolism markers are shown. ALP: alkaline phosphatase, BAP: bone-specific alkaline phosphatase, Ca: calcium, Cre: creatinine, P: phosphate, PTH: parathyroid hormone, TRACP5b: tartrate-resistant acid phosphatase isoform $5 b$

zyme inhibitors or angiotensin receptor blockers, were prescribed.

On admission, the patient could not move his body because of severe thoracic, low back, hip joint, and foot joint pain. Initial laboratory tests revealed the presence of severe hypocalcemia with a low serum 25-hydroxyvitamin D level (11 ng/mL), elevated serum ALP, bone-specific alkaline phosphatase (BAP), tartrate-resistant acid phosphatase isoform 5b (TRACP5b), and intact parathyroid hormone (PTH) levels (Fig. 3). His serum creatinine and urea nitrogen levels were $9.95 \mathrm{mg} / \mathrm{dL}$ and $79 \mathrm{mg} / \mathrm{dL}$, respectively. Electrocardiography showed QT prolongation (QTc, $508 \mathrm{~ms}$ ). His lumbar spine T-score of -2.8 and femoral neck T-score of -3.7 on admission indicated that his BMD had worsened (Fig. 2B). On bone scintigraphy, the accumulation of technetium-99 m hydroxymethylene diphosphonate $\left({ }^{99 \mathrm{~m}} \mathrm{Tc}-\right.$ HMDP) was observed in the ribs and at the distal end of the right tibia, although a radiographic examination of the corresponding area showed no signs of fracture (Fig. 4A and B). These findings indicated severe osteomalacia. A diagnostic interview on admission revealed poor medication adherence; the patient had rarely taken the prescribed calcitriol. Thus, we started medication and peritoneal dialysis, as shown in Fig. 3A, while paying careful attention to his medication adherence. Several reports have shown the necessity of native vitamin D supplementation to improve the bone status of patients with osteomalacia and end-stage renal disease. We therefore used cholecalciferol in addition to falecalcitriol and eldecalcitol $(7,8)$. These therapies gradually corrected his serum calcium level (Fig. 3A). Consequently, his serum ALP, BAP, and TRACP5b levels temporarily increased (Fig. 3B). At 7 and 15 months after admission, the patient's BMD had improved (Fig. 2B). At 10 months after admission, no accumulation of ${ }^{99 \mathrm{~m}} \mathrm{Tc}-\mathrm{HMDP}$ was observed on bone scintigraphy (Fig. 4A). The urinary calcium (mg/dL)/ creatinine $(\mathrm{mg} / \mathrm{dL})$ ratios during the treatment period ranged from 0.049 to 0.108 .

Abdominal computed tomography (CT) on admission showed nephrocalcinosis (Fig. 4C). Based on the patient's symptoms, a diagnosis of Dent disease was suggested. We therefore obtained informed consent according to the policy of Tokyo Medical and Dental University to perform comprehensive genetic diagnostic testing using a next-generation sequencing (NGS) panel. The NGS panel showed that the 

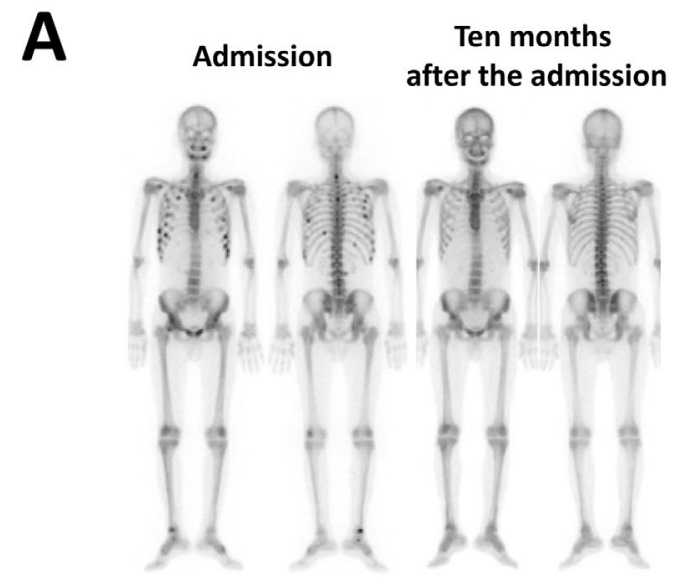

Anterior Posterior Anterior Posterior
B

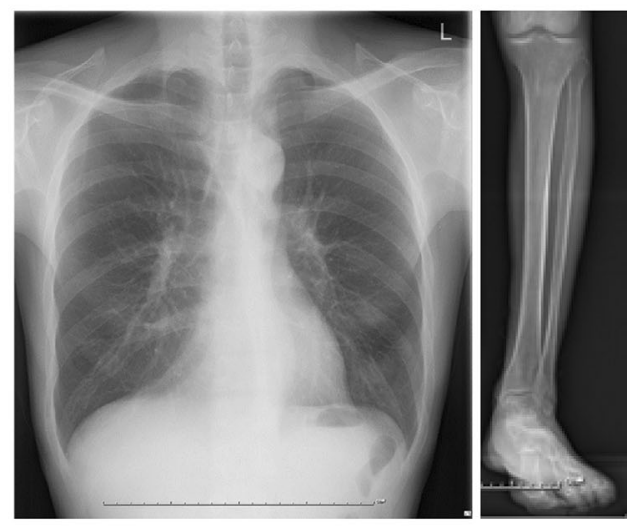

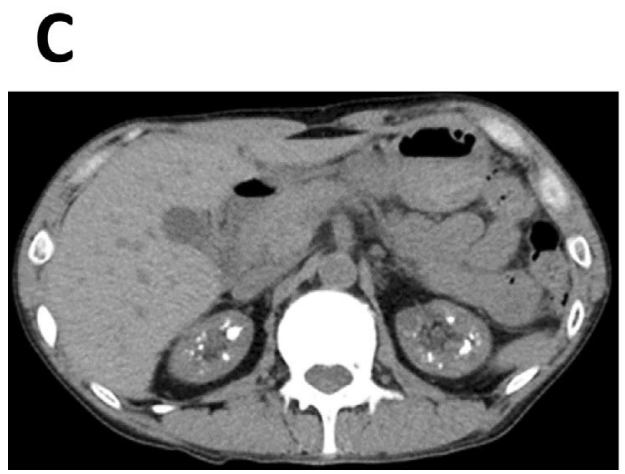

Figure 4. Clinical images of the current case. (A) Bone scintigraphy on admission showed the accumulation of technetium-99m hydroxymethylene diphosphonate $\left({ }^{99} \mathrm{~m}\right.$ Tc-HMDP) at multiple sites. Tracer accumulation was not observed after treatment. (B) A radiographic examination on admission showed no signs of fracture. (C) Nephrocalcinosis was evident on abdominal computed tomography.

patient had an intronic mutation $(1348-1 \mathrm{G}>\mathrm{A})$ of the CLCN5 gene (Fig. 5A) (9). Even though the NGS panel covered other genes responsible for hereditary hypocalcemia (GNA11, STX16, GNAS-AS1, GNAS, PTH, GCM2) and Fanconi syndrome (LC34A1, CA2, EHHADH, HNF4A, $S L C 2 A 2)$, no causative variants were detected in those genes. The mutation $(1348-1 \mathrm{G}>\mathrm{A})$ of the CLCN5 gene has not been listed in the Human Gene Mutation Database (HGMD) (http://www.hgmd.cf.ac.uk/ac/index.php) or in ClinVar, a public archive of the relationships among human variations and phenotypes (https://www.ncbi.nlm.nih.gov/clin var/), indicating that the $1348-1 \mathrm{G}>\mathrm{A}$ mutation was a previously unrecognized mutation. We also searched genomic databases of healthy subjects, including ExAC (http://exac. broadinstitute.org/), the International Genome Sample Resource (IGSR) (http://www.internationalgenome.org), the Exome Variant Server (http://evs.gs.washington.edu/EVS/), the Human Genetic Variation Database (http://www.hgvd.gen ome.med.kyoto-u.ac.jp/), and the Japanese Genome Variation Database (2KJPN) (https://ijgvd.megabank.tohoku.ac.jp/); the $1348-1 \mathrm{G}>\mathrm{A}$ mutation was not found in any of these genomic databases. Because the mutation was located just prior to the first base pair of exon 9, we investigated the effects of the intronic mutation on the splicing of CLCN5 messenger RNA (mRNA). Sequencing analyses of mRNA obtained from peripheral blood leukocytes revealed that the first 26 bases of exon 9 were spliced out in this patient (Fig. 5B). This 26-base deletion in the mRNA of CLCN5 results in a translational frameshift and C-terminal truncation of $\mathrm{ClC}-5$. As a result of this truncation, ClC-5 in the current case loses its cystathionine-beta-synthase (CBS) domains (Fig. 5C). Computational prediction using the Iterative Threading ASSEmbly Refinement (I-TASSER) program (Zhang Labs, University of Kansas, Lawrence, USA) revealed that the mutation in the current case causes not only C-terminal truncation, but also a structural change of the 3dimensional architecture of the N-terminal region of $\mathrm{ClC}-5$ (Protein Data Bank, PDB ID 2J9L; Fig. 5D) (10-12). We further analyzed whether the mutation functionally impairs ClC-5. We obtained proximal tubule cells from the patient's urine. The origin of the cultured cells was confirmed by immunocytochemistry for megalin. Anti-megalin antibody, which was used at 1:2,000 dilution, was provided by Dr. T. Michigami, Osaka Medical Center and Research Institute for Maternal and Child Health, Osaka, Japan (Fig. 6) (13). Normal rat kidney 49F (NRK49F) cells served as a negative control for megalin staining. Using pHrodo, which is an indicator of endosomal and/or lysosomal acidification, we 

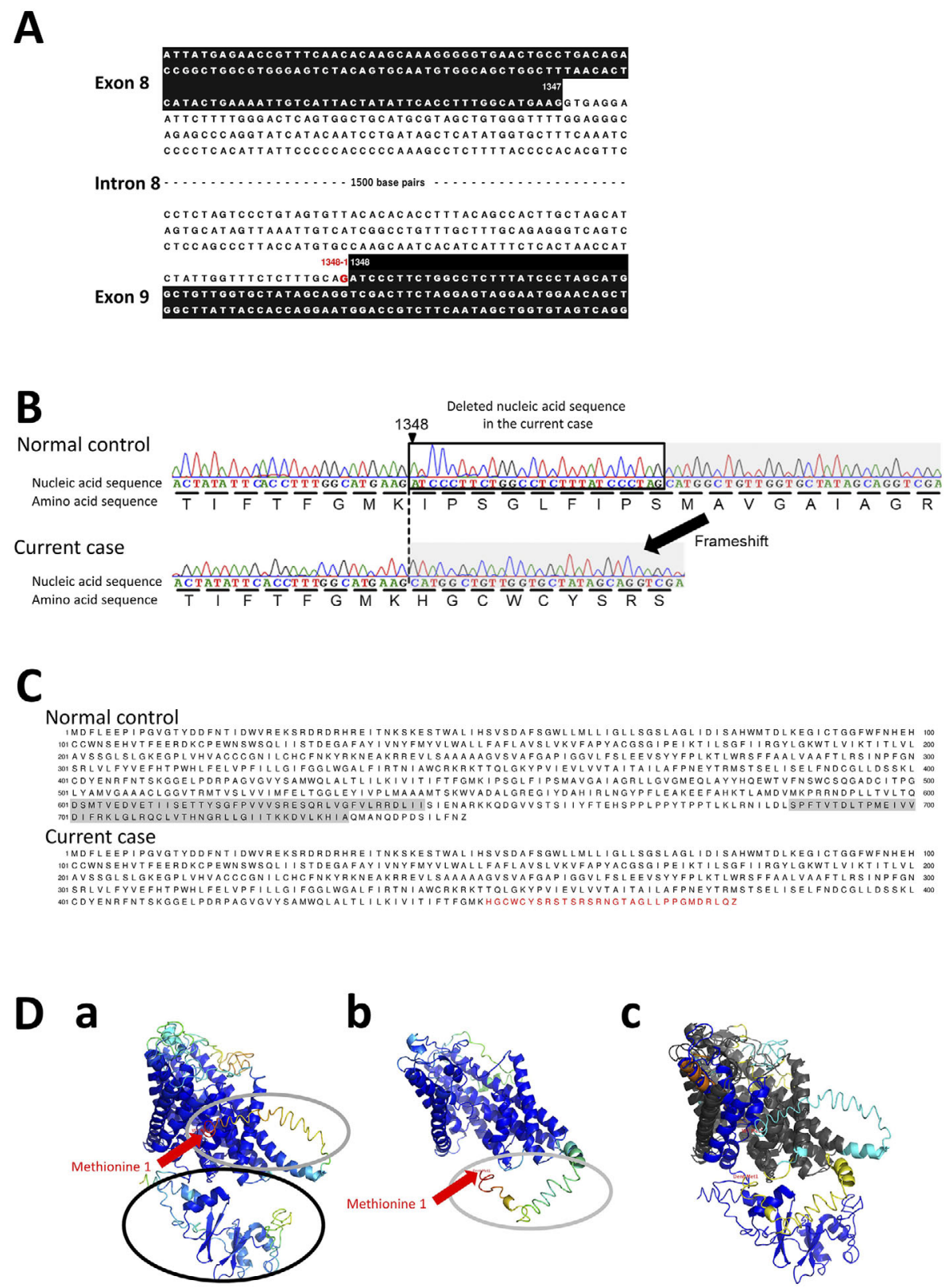

Figure 5. A previously unrecognized intronic mutation of the CLCN5 gene caused the patient's Dent disease. (A) Genomic DNA sequencing revealed that the last base of intron 8 (shown in red) was mutated to adenine (1348-1 G>A). (B) Messenger RNA sequencing showed that the first 26 bases of exon 9 were spliced out following the AT-AG rule of intron. RNA was obtained from the peripheral blood leukocytes. (C) The amino acid sequences of $\mathrm{H} \mathrm{(+)} \mathrm{/Cl} \mathrm{(-)} \mathrm{exchange} \mathrm{transporter} 5$ (CIC-5) are shown. A gray background indicates the cystathionine-beta-synthase (CBS) domains. Red indicates amino acid mutations due to the frameshift. The C-terminal portion of ClC-5, which contains the CBS domains, was truncated in the current case. (D) Computational predictions of the 3-dimensional architecture of wild-type ClC-5 (a) and the current case (b) are shown. The structures were calculated using the I-TASSER program and colored by B-factor, which indicates the thermal mobility of residues in proteins. Red-colored residues in N-termini (gray ellipses) have a high B-factor. The black ellipse indicates the CBS domain. (c) A merged image of wild-type CIC-5 and the current case is shown. The black structure indicates the aligned residue pairs, whereas cyan and yellow indicate nonaligned residues in wild-type $\mathrm{CIC}-5$ and the current case, respectively. The orange structure indicates the mutated portion of $\mathrm{ClC}-5$ in the current case. The truncated portion of $\mathrm{ClC}-5$ in the current case is shown in blue. 

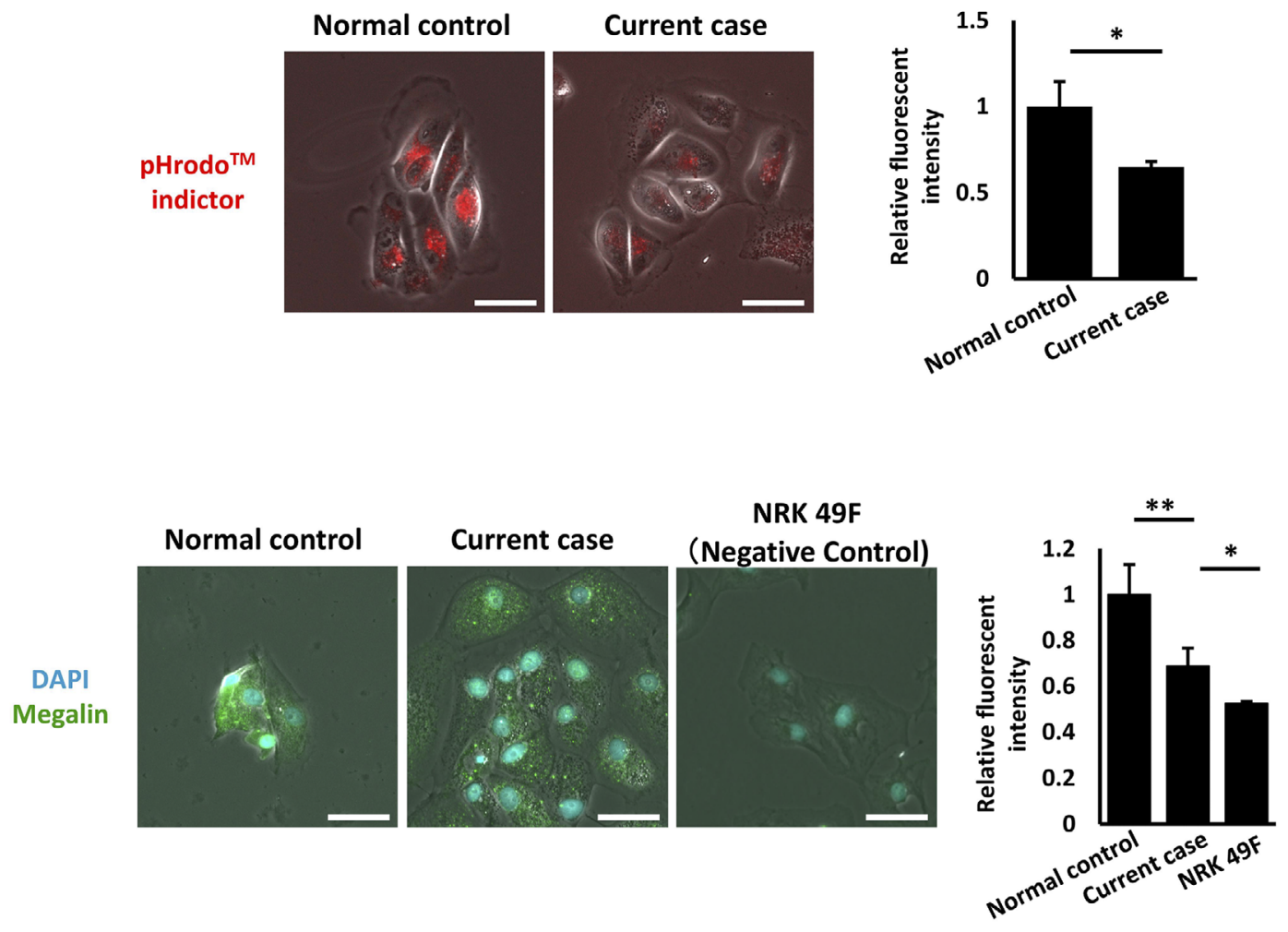

Figure 6. Acidification of the endosomes and/or lysosomes was impaired in the cultured proximal tubule cells of the current case. (A) Acidification of the endosomes and/or lysosomes was observed in cultured proximal tubule cells. Cloned urinary cells were cultured in Dulbecco's Modified Eagle's Medium and Ham's F-12 Nutrient Mixture (DMEM/F12) supplemented with insulin, transferrin, selenium, ethanolamine (ITS-X), and $\mathbf{1 0 \%}$ fetal calf serum. Endosomal and/or lysosomal acidification was visualized using pHrodo. A red signal indicates acidified organelle. Immunocytochemical staining for megalin (green) confirmed that these cells were proximal tubule cells. Normal rat kidney 49F (NRK49F) cells served as a negative control for megalin staining (scale bar: $50 \mu \mathrm{m})(\mathrm{n}=\mathbf{4 - 7}$ in each group: ${ }^{*} \mathbf{p}<\mathbf{0 . 0 5}$, **p<0.01, t-test for $\mathbf{p H r o d o}$ and Dunnett's test for megalin staining). Relative fluorescent intensities were analyzed using the Image $\mathbf{J}$ software program.

found that acidification was impaired in the patient's proximal tubule cells (Fig. 6). The intensity of megalin staining was lower in comparison to a normal control (Fig. 6).

\section{Discussion}

Several factors might have contributed to the development of severe osteomalacia in the current case. First, impaired reabsorption of phosphate by the proximal tubule as a result of Fanconi syndrome might have been a contributing factor. The finding of hypophosphatemia and a low BMD at 137 to 50 months prior to admission supports the diagnosis of Fanconi syndrome, and this might have contributed to the pathogenesis of the osteomalacia. Second, hypocalcemia secondary to the impaired activation of vitamin D as a result of progressive renal dysfunction, added to the effects of hypophosphatemia during the 4 years prior to admission. The steep decline in BMD during this 4 years indicates that not only hypophosphatemia, but also hypocalcemia, contributed to the pathogenesis. The patient's serum 1,25dihydroxyvitamin D level on admission was around the lower limit of normal $(26 \mathrm{pg} / \mathrm{mL})$. Because he had taken calcitriol for several days just before admission, his serum 1,25-dihydroxyvitamin D level without oral calcitriol might have been much lower. Third, vitamin D deficiency also contributed to the pathogenesis. Although 25hydroxyvitamin D is not an active form of vitamin D, several reports have shown that native vitamin D supplementation was required to improve the bone status in patients with osteomalacia and end-stage renal disease $(7,8)$. As the patient had severe pain in the months prior to admission, he had remained largely indoors. A lack of sunlight exposure might have contributed to the reduced serum 25hydroxyvitamin D level at admission. Fourth, poor medication adherence contributed to the pathogenesis. The diagnostic interview on admission revealed that the patient had rarely taken the prescribed calcitriol because he was anxious about his serum creatinine level, which had increased at the initiation of calcitriol therapy. Although calcium and vitamin D overload should be avoided in patients with Dent disease, the fine-tuning of the calcium/phosphate balance should have been carried out with appropriate medication adherence.

We successfully treated this patient's osteomalacia. All pa- 
rameters, including the serum calcium level, BMD, and tracer accumulation on bone scintigraphy, dramatically improved. The patient's serum ALP, BAP, and TRACP5b levels temporarily increased following the elevation of his serum calcium level. Because extracellular calcium has been reported to increase the expression of bone morphogenetic protein 2, an inducer of osteoblastic differentiation, the elevated serum calcium level in this patient might have caused the elevation of his ALP and BAP levels (14). The subsequent decrease in his ALP and BAP levels reflects accomplished osteocyte differentiation and bone mineralization (15). The temporary increase in the TRACP5b level during the therapeutic period might also reflect an improved osteocyte function. Osteocytes, under stimulation of PTH, upregulate the expression of receptor activator for nuclear factor- $\kappa \mathrm{B}$ ligand (RANKL), which binds the RANK expressed on osteoclast precursors, thereby activating the signaling pathways that promote osteoclast differentiation (16).

The prevalence of osteomalacia is extremely low in Japanese patients with the CLCN5 mutation (5). While the vitamin D status within different European populations is variable, the overall prevalence of hypovitaminosis D in Europe is higher than that in Japan (17). Thus, the vitamin D status may explain why the prevalence of osteomalacia in Japanese patients with CLCN5 mutations is lower than that in European patients. A lower latitude and higher consumption of fish may result in the better vitamin D status in Japan. Indeed, Nakamura et al. showed that Japanese individuals who frequently consumed fish had higher serum 25hydroxyvitamin D concentrations, especially in winter (18).

Although it has been shown that $\mathrm{ClC}-5$ plays an essential role in endosomal acidification in the kidney, the precise mechanism as to how impaired endosomal acidification causes renal dysfunction remains uncertain. Recently, Blanchard et al. reported the characteristics of a large cohort of patients with Dent disease (19). They showed that although the renal failure in Dent disease is frequently attributed to nephrocalcinosis, the rate of decrease in the estimated glomerular filtration rate (eGFR) with age did not differ according to the presence or absence of nephrocalcinosis (19). They also showed that there was no difference between severe and moderate mutations of the CLCN5 gene with regard to the age at the diagnosis, eGFR, proteinuria, and calciuria (19). Thus, nephrocalcinosis and/or severity in the CLCN5 mutation may not directly influence the progression of renal dysfunction. Blanchard et al. also reported that proteinuria apparently worsened with age, and that more than half of patients of $\geq 18$ years of age had proteinuria $>2$ $\mathrm{g} /$ day (19). As proteinuria is an established prognostic indicator in the progression of chronic kidney disease, persistent proteinuria may cause-at least in part-renal dysfunction in patients with Dent disease.

A previously unrecognized intronic mutation, 1348-1G $>$ A, was found in the current case. Following the GT-AG rule, in which intron junctions start with the dinucleotide GT and end with AG, the intronic mutation $1348-1 \mathrm{G}>\mathrm{A}$ causes a $26-$ base deletion in CLCN5 mRNA. As a result of the translational frameshift, the ClC-5 protein loses its CBS domains. The importance of the CBS domains in the function of $\mathrm{ClCs}$ has been confirmed by a series of mutations in other members of the $\mathrm{ClC}$ family proteins. For example, point mutations in the CBS domains in ClC-1, CIC-2, CIC-7 and CIC$\mathrm{Kb}$ result in myotonia, idiopathic generalized epilepsy, osteopetrosis, and Bartter syndrome, respectively (20). A computational analysis showed that the mutation in the current case caused not only $\mathrm{C}$-terminal truncation, but also a structural change in the 3-dimensional architecture of the $\mathrm{N}$ terminal region of $\mathrm{ClC}-5$. Although we have not experimentally confirmed whether $1348-1 \mathrm{G}>\mathrm{A}$ causes this structural change, the impaired acidification in the cultured proximal tubule cells supports the hypothesis that the 1348-1G>A mutation is a cause of Dent disease.

The authors state that they have no Conflict of Interest (COI).

\section{References}

1. van Berkel Y, Ludwig M, van Wijk JAE, Bökenkamp A. Proteinuria in Dent disease: a review of the literature. Pediatr Nephrol 32: 1851-1859, 2017.

2. Böckenhauer D, Bökenkamp A, Nuutinen M, et al. Novel OCRL mutations in patients with Dent-2 disease. J Pediatr Genet 1: 1523, 2012.

3. Devuyst O, Christie PT, Courtoy PJ, Beauwens R, Thakker RV. Intra-renal and subcellular distribution of the human chloride channel, CLC-5, reveals a pathophysiological basis for Dent's disease. Hum Mol Genet 8: 247-257, 1999.

4. Claverie-Martín F, Ramos-Trujillo E, García-Nieto V. Dent's disease: clinical features and molecular basis. Pediatr Nephrol 26: 693-704, 2011.

5. Sekine T, Komoda F, Miura K, et al. Japanese Dent disease has a wider clinical spectrum than Dent disease in Europe/USA: genetic and clinical studies of 86 unrelated patients with low-molecularweight proteinuria. Nephrol Dial Transplant 29: 376-384, 2014.

6. Isakova T, Wahl P, Vargas GS, et al. Fibroblast growth factor 23 is elevated before parathyroid hormone and phosphate in chronic kidney disease. Kidney Int 79: 1370-1378, 2011.

7. Mac-Way F, Azzouz L, Noel C, Lafage-Proust MH. Osteomalacia induced by vitamin $\mathrm{D}$ deficiency in hemodialysis patients: the crucial role of vitamin D correction. J Bone Miner Metab 32: 215219,2014

8. Hernandez JD, Wesseling K, Boechat MI, Gales B, Salusky IB. Osteomalacia in a hemodialysis patient receiving an active vitamin D sterol. Nat Clin Pract Nephrol 3: 227-232, 2007.

9. Mori T, Hosomichi K, Chiga M, et al. Comprehensive genetic testing approach for major inherited kidney diseases, using nextgeneration sequencing with a custom panel. Clin Exp Nephrol 21: 63-75, 2017.

10. Roy A, Kucukural A, Zhang Y. I-TASSER: a unified platform for automated protein structure and function prediction. Nat Protoc 5: 725-738, 2010.

11. Yang J, Yan R, Roy A, Xu D, Poisson J, Zhang Y. The I-TASSER suite: protein structure and function prediction. Nat Methods 12: 7-8, 2014.

12. Zhang Y. I-TASSER server for protein $3 \mathrm{D}$ structure prediction. BMC Bioinformatics 9: 1-8, 2008.

13. Yamagata M, Ozono K, Hashimoto Y, Miyauchi Y, Kondou H, Michigami T. Intraperitoneal administration of recombinant 
receptor-associated protein causes phosphaturia via an alteration in subcellular distribution of the renal sodium phosphate cotransporter. J Am Soc Nephrol 16: 2338-2345, 2005.

14. Tada H, Nemoto E, Kanaya S, Hamaji N, Sato H, Shimauchi H. Elevated extracellular calcium increases expression of bone morphogenetic protein-2 gene via a calcium channel and ERK pathway in human dental pulp cells. Biochem Biophys Res Commun 394: 1093-1097, 2010.

15. Kato Y, Boskey A, Spevak L, Dallas M, Hori M, Bonewald LF Establishment of an osteoid preosteocyte-like cell MLO-A5 that spontaneously mineralizes in culture. J Bone Miner Res 16: 1622 1633, 2001.

16. Boyce BF, Xing L. Biology of RANK, RANKL, and osteoprotegerin. Arthritis Res Ther 9(Suppl 1): S1, 2007.

17. Mithal A, Wahl DA, Bonjour JP, et al. Global vitamin D status and determinants of hypovitaminosis D. Osteoporos Int 20: 1807-
1820, 2009.

18. Nakamura K, Nashimoto M, Matsuyama S, Yamamoto M. Low serum concentrations of 25-hydroxyvitamin D in young adult Japanese women: a cross sectional study. Nutrition 17: 921-925, 2001.

19. Blanchard A, Curis E, Guyon-Roger T, et al. Observations of a large Dent disease cohort. Kidney Int 90: 430-439, 2016.

20. Ignoul S, Eggermont J. CBS domains: structure, function, and pathology in human proteins. Am J Physiol - Cell Physiol 289: C1369-C1378, 2005.

The Internal Medicine is an Open Access journal distributed under the Creative Commons Attribution-NonCommercial-NoDerivatives 4.0 International License. To view the details of this license, please visit (https://creativecommons.org/licenses/ by-nc-nd/4.0/).

(C) 2018 The Japanese Society of Internal Medicine Intern Med 57: 3603-3610, 2018 